Table 2 (continued)

\begin{tabular}{|c|c|c|c|}
\hline Region/Country & 1982 & 1985 & 1988 \\
\hline $\begin{array}{l}\text { Qatar } \\
\text { Saudi Arabia } \\
\text { Senegal } \\
\text { Sierre Leone } \\
\text { South Africa } \\
\text { Sudan } \\
\text { Tanzania } \\
\text { Togo } \\
\text { Tunisia } \\
\text { Uganda } \\
\text { Zaire } \\
\text { Zambia } \\
\text { Zimbabwe }\end{array}$ & $\begin{array}{l}1 \\
2 \\
0 \\
0 \\
0 \\
0 \\
2 \\
1 \\
0 \\
1 \\
1 \\
0 \\
2\end{array}$ & $\begin{array}{l}0 \\
1 \\
1 \\
0 \\
2 \\
0 \\
2 \\
0 \\
1 \\
0 \\
0 \\
1 \\
3\end{array}$ & $\begin{array}{l}0 \\
0 \\
1 \\
1 \\
4 \\
0 \\
0 \\
0 \\
2 \\
1 \\
0 \\
0 \\
1\end{array}$ \\
\hline Asia & 65 & 124 & 160 \\
\hline $\begin{array}{l}\text { Bangladesh } \\
\text { Burma } \\
\text { China } \\
\text { Hong Kong } \\
\text { India } \\
\text { Indonesia } \\
\text { Japan } \\
\text { Korea } \\
\text { Malaysia } \\
\text { Pakistan } \\
\text { Singapore } \\
\text { Sri Lanka } \\
\text { Taiwan } \\
\text { Thailand }\end{array}$ & $\begin{array}{r}0 \\
0 \\
0 \\
0 \\
30 \\
0 \\
20 \\
13 \\
0 \\
0 \\
1 \\
1 \\
0 \\
0\end{array}$ & $\begin{array}{r}1 \\
0 \\
11 \\
4 \\
66 \\
0 \\
21 \\
12 \\
1 \\
1 \\
1 \\
1 \\
1 \\
3\end{array}$ & $\begin{array}{r}8 \\
1 \\
19 \\
3 \\
67 \\
1 \\
25 \\
18 \\
0 \\
0 \\
3 \\
1 \\
12 \\
2\end{array}$ \\
\hline Unknown & & & 82 \\
\hline Total & 1,477 & 1,763 & $1,608^{* * *}$ \\
\hline
\end{tabular}

"nk indicates exact number, if any, are "not known."

* Note: The Paris Congress Participation figures are inflated by the inclusion of 56 press, 110 exhibitors and an unknown number of individuals accompanying the participants. If exhibitors were added to the Washington Congress figures the total participants would increase by 353 , to 1961. The figure of 1608 represents the total number of participants.

\title{
Participation by Women in the 1988 IPSA Meeting
}

Martin Gruberg

University of Wisconsin

Since 1972 the author has monitored participation by women at the annual meetings of the American Political Science Association. His annual assessments appearing in PS have documented the emergence of American female political scientists from being rarities to 
their present manifestation as about $20 \%$ of the APSA program participants. I thought it would be useful to make a similar survey of the participation of women at the 1988 IPSA meeting.

Though the overall totals are not as dismal as an initial impression of the program might suggest, in fact, if not for mutual help strategies by women, female presence would be much more of a rarity.

Participation in the Plenary Sessions, Subfield Sections, Research Committees, Study Groups, Special Sessions, and Current Research Sessions

Research Sessions

Organizers

Chairpersons

Paper Givers

Discussants

Total Women \% Total Women \% Total Women \% Total Women \%

$\begin{array}{llllllllllll}90 & 14 & 15.6 & 252 & 34 & 13.5 & 1.060 & 150 & 14.2 & 289 & 56 & 19.4\end{array}$

In Sections Organized by Women

$\begin{array}{lllllllll}40 & 18 & 14.5 & 192 & 55 & 28.6 & 60 & 21 & 35.0\end{array}$

In Panels Organized by Women

$\begin{array}{llllll}107 & 42 & 39.3 & 28 & 16 & 57.1\end{array}$

In Panels Dealing with Status of Women

$\begin{array}{lllllllll}10 & 9 & 90.0 & 35 & 31 & 88.6 & 14 & 12 & 85.7\end{array}$

Though not always true, where women serve as section heads or chairpersons, this usually facilitates opportunities for other women. The old boys' network often has a blind spot in recognizing that there are indeed women making contributions to the many fields of political science. This is especially true at international meetings. Many countries have a small number of female political scientists. Those women who are members of the profession are not apt to teach at major universities or to be in senior positions. They face greater difficulties in getting funding to travel to IPSA meetings. Males are the gatekeepers.

It is sometimes argued that there are not women doing research in some areas of the discipline. To refute this defense, I've extracted from the 1988 APSA and IPSA programs data on participation rates in some of the subjects. (Inasmuch as the two organizations structured their meetings differently, there is only an approximate opportunity for paralleling the information.)

Political Methodology

(Miniplenary I and Research Committee 7:

Section 4 and Organized Section on Political Methodology)

\begin{tabular}{|c|c|c|c|c|c|c|c|c|}
\hline \multicolumn{3}{|c|}{ Chairpersons } & \multicolumn{3}{|c|}{ Paper Givers } & \multicolumn{3}{|c|}{ Discussants } \\
\hline Total & Women & $\%$ & Total & Women & $\%$ & Total & Women & $\%$ \\
\hline IPSA & & & & & & & & \\
\hline 5 & 0 & 0 & 13 & 3 & 23.1 & 2 & 0 & 0 \\
\hline APSA & & & & & & & & \\
\hline 11 & 1 & 9.1 & 60 & 14 & 23.3 & 13 & 0 & 0 \\
\hline
\end{tabular}


Political Theory

(Subfields I and II and Research Committee 31;

Sections 1, 2. and 3 and Organized Section on Foundation of Political Theory)

\begin{tabular}{|c|c|c|c|c|c|c|c|c|}
\hline \multicolumn{3}{|c|}{ Chairpersons } & \multicolumn{3}{|c|}{ Paper Givers } & \multicolumn{3}{|c|}{ Discussants } \\
\hline Total & Women & $\%$ & Total & Women & $\%$ & Total & Women & $\%$ \\
\hline \multicolumn{9}{|l|}{ IPSA } \\
\hline 8 & 0 & 0 & 35 & 1 & 2.9 & 7 & 0 & 0 \\
\hline \multicolumn{9}{|l|}{ APSA } \\
\hline 33 & 7 & 21.2 & 111 & 22 & 19.8 & 50 & 8 & 16.0 \\
\hline \multicolumn{9}{|c|}{$\begin{array}{l}\text { Political Economy } \\
\text { (Subfield III; Section II) }\end{array}$} \\
\hline \multicolumn{3}{|c|}{ Chairpersons } & \multicolumn{3}{|c|}{ Paper Givers } & \multicolumn{3}{|c|}{ Discussants } \\
\hline Total & Women & $\%$ & Total & Women & $\%$ & Total & Women & $\%$ \\
\hline \multicolumn{9}{|l|}{ IPSA } \\
\hline 3 & 1 & 16.7 & 23 & 1 & 4.3 & 6 & 2 & 33.3 \\
\hline \multicolumn{9}{|l|}{ APSA } \\
\hline 7 & 1 & 14.3 & 30 & 9 & 3.0 & 8 & 1 & 12.5 \\
\hline
\end{tabular}

International Security

(Subfield IV and Research Committee 24;

Sections 24 and 25 and Organized Section on Conflict Processes)

Chairpersons

Paper Givers

Discussants

\begin{tabular}{rccccccccc}
$\begin{array}{c}\text { Total } \\
\text { IPSA }\end{array}$ & Women & $\%$ & Total & Women & $\%$ & Total & Women & $\%$ \\
12 & 1 & 8.3 & 55 & 6 & 10.9 & 12 & 2 & 16.7 \\
$\begin{aligned} \text { APSA } \\
27\end{aligned}$ & 2 & 7.4 & 114 & 16 & 13.8 & 33 & 6 & 18.2 \\
\hline \multicolumn{8}{c}{$\begin{array}{c}\text { Foreign Policy Analysis } \\
\text { (Subfieid V: Section 27) }\end{array}$}
\end{tabular}

\begin{tabular}{rcccccccc}
\multicolumn{2}{c}{ Chairpersons } & \multicolumn{3}{c}{ Paper Givers } & \multicolumn{3}{c}{ Discussants } \\
Total & Women & $\%$ & Total & Women & $\%$ & Total & Women & $\%$ \\
IPSA & 1 & 25.0 & 18 & 0 & 0 & 4 & 1 & 25.0 \\
4 & 3 & 37.5 & 33 & 5 & 15.2 & 7 & 2 & 28.6 \\
$\begin{array}{r}\text { APSA } \\
8\end{array}$ & & & & & & & & \\
\hline
\end{tabular}

Comparative National Institutions

(Subfield Vi: Section 9)

\begin{tabular}{|c|c|c|c|c|c|c|c|c|}
\hline \multicolumn{3}{|c|}{ Chairpersons } & \multicolumn{3}{|c|}{ Paper Givers } & \multicolumn{3}{|c|}{ Discussants } \\
\hline Total & Women & $\%$ & Total & Women & $\%$ & Total & Women & $\%$ \\
\hline IPSA & & & & & & & & \\
\hline 5 & 0 & 0 & 23 & 4 & 17.4 & 8 & 0 & 0 \\
\hline APSA & & & & & & & & \\
\hline 7 & 0 & 0 & 28 & 4 & 14.3 & 10 & 1 & 10.0 \\
\hline
\end{tabular}




\section{Participation by Women}

\begin{tabular}{|c|c|c|c|c|c|c|c|c|}
\hline \multirow{2}{*}{\multicolumn{3}{|c|}{ Chairpersons }} & \multicolumn{3}{|c|}{ Development } & \multirow{2}{*}{\multicolumn{3}{|c|}{ Discussants }} \\
\hline & & & \multicolumn{3}{|c|}{ Paper Givers } & & & \\
\hline Total & Women & $\%$ & Total & Women & $\%$ & Total & Women & $\%$ \\
\hline \multicolumn{9}{|c|}{ IPSA (Development and Political System) } \\
\hline 1 & 0 & 0 & 6 & 0 & 0 & - & - & - \\
\hline \multicolumn{9}{|c|}{ APSA (Developing Areas and the Internationa! System) } \\
\hline 8 & 3 & 37.5 & 25 & 9 & 36.0 & 9 & 2 & 22.2 \\
\hline \multicolumn{9}{|c|}{ Development } \\
\hline \multicolumn{3}{|c|}{ Chairpersons } & \multicolumn{3}{|c|}{ Paper Givers } & \multicolumn{3}{|c|}{ Discussants } \\
\hline Total & Women & $\%$ & Total & Women & $\%$ & Total & Women & $\%$ \\
\hline \multicolumn{9}{|c|}{ IPSA (Women, Politics and Development) } \\
\hline 1 & 1 & 100 & 4 & 4 & 100 & 2 & 2 & 100 \\
\hline \multicolumn{9}{|c|}{ APSA (Comparative Politics of Developing Areas) } \\
\hline 7 & 0 & 0 & 29 & 8 & 20.6 & 12 & 1 & 8.0 \\
\hline
\end{tabular}

Political Parties

(Subfield VIl; Section 20 and

Organized Section on Political Organizations and Parties)

\begin{tabular}{|c|c|c|c|c|c|c|c|c|}
\hline \multicolumn{3}{|c|}{ Chairpersons } & \multicolumn{3}{|c|}{ Paper Givers } & \multicolumn{3}{|c|}{ Discussants } \\
\hline Total & Women & $\%$ & Total & Women & $\%$ & Total & Women & $\%$ \\
\hline \multicolumn{9}{|l|}{ IPSA } \\
\hline 10 & 0 & 0 & 32 & 3 & 9.4 & 9 & 3 & 33.3 \\
\hline APSA & & & . & & & & & \\
\hline 14 & 5 & 35.7 & 62 & 8 & 12.9 & 12 & 3 & 2.5 \\
\hline \multicolumn{9}{|c|}{$\begin{array}{c}\text { Election Systems } \\
\text { (Study Group 24: Section 19) }\end{array}$} \\
\hline \multicolumn{3}{|c|}{ Chairpersons } & \multicolumn{3}{|c|}{ Paper Givers } & \multicolumn{3}{|c|}{ Discussants } \\
\hline Total & Women & $\%$ & Total & Women & $\%$ & Total & Women & $\%$ \\
\hline IPSA & & & & & & & & \\
\hline 3 & 1 & 33.3 & 15 & 1 & 6.7 & 6 & 2 & 33.3 \\
\hline \multicolumn{9}{|l|}{ APSA } \\
\hline 13 & 3 & 23.1 & 63 & 8 & 12.9 & 14 & 3 & 21.4 \\
\hline \multicolumn{9}{|c|}{$\begin{array}{c}\text { Political Attitudes and Participation } \\
\text { (Subfield VIII; Section 16) }\end{array}$} \\
\hline \multicolumn{3}{|c|}{ Chairpersons } & \multicolumn{3}{|c|}{ Paper Givers } & \multicolumn{3}{|c|}{ Discussants } \\
\hline Total & Women & $\%$ & Total & Women & $\%$ & Total & Women & $\%$ \\
\hline \multicolumn{9}{|l|}{ IPSA } \\
\hline 13 & 3 & 23.1 & 57 & 10 & 17.5 & 18 & 4 & 22.2 \\
\hline \multicolumn{9}{|l|}{ APSA } \\
\hline 8 & 1 & 12.5 & 45 & 5 & 11.1 & 7 & 0 & 0 \\
\hline
\end{tabular}




\section{Local Government}

(Subfield IX, Research Committee 5;

Section 21 and Organized Section on Urban Politics)

\begin{tabular}{|c|c|c|c|c|c|c|c|c|}
\hline \multicolumn{3}{|c|}{ Chairpersons } & \multicolumn{3}{|c|}{ Paper Givers } & \multicolumn{3}{|c|}{ Discussants } \\
\hline Total & Women & $\%$ & Total & Women & $\%$ & Total & Women & $\%$ \\
\hline IPSA & & & & & & & & \\
\hline 10 & 2 & 20.0 & 45 & 4 & 8.9 & 17 & 2 & 17.6 \\
\hline \multicolumn{9}{|l|}{ APSA } \\
\hline 19 & 2 & 10.5 & 76 & 10 & 13.2 & 24 & 5 & 20.8 \\
\hline \multicolumn{9}{|c|}{$\begin{array}{c}\text { Public Administration } \\
\text { (Subfield X; Organized Section on Public Administration) }\end{array}$} \\
\hline \multicolumn{3}{|c|}{ Chairpersons } & \multicolumn{3}{|c|}{ Paper Givers } & \multicolumn{3}{|c|}{ Discussants } \\
\hline Total & Women & $\%$ & Total & Women & $\%$ & Total & Women & $\%$ \\
\hline IPSA & & & & & & & & \\
\hline 4 & 1 & 25.0 & 19 & 5 & 26.3 & 4 & 2 & 50.0 \\
\hline \multicolumn{9}{|l|}{ APSA } \\
\hline 12 & 5 & 41.7 & 49 & 12 & 24.5 & 15 & 5 & 33.3 \\
\hline \multicolumn{9}{|c|}{$\begin{array}{l}\text { Bureaucracy } \\
\text { (Study Group 27; Section 18) }\end{array}$} \\
\hline \multicolumn{3}{|c|}{ Chairpersons } & \multicolumn{3}{|c|}{ Paper Givers } & \multicolumn{3}{|c|}{ Discussants } \\
\hline Total & Women & $\%$ & Total & Women & $\%$ & Total & Women & $\%$ \\
\hline \multicolumn{9}{|l|}{ IPSA } \\
\hline 2 & 0 & 0 & 10 & 0 & 0 & 4 & 1 & 25.0 \\
\hline \multicolumn{9}{|l|}{ APSA } \\
\hline 7 & 3 & 42.9 & 31 & 7 & 22.6 & 9 & 4 & 44.4 \\
\hline
\end{tabular}

\section{Legislatures}

(Research Committee 8; Section 13 and

Organized Section on Legislative Studies)

\begin{tabular}{|c|c|c|c|c|c|c|c|c|}
\hline \multicolumn{3}{|c|}{ Chairpersons } & \multicolumn{3}{|c|}{ Paper Givers } & \multicolumn{3}{|c|}{ Discussants } \\
\hline Total & Women & $\%$ & Total & Women & $\%$ & Total & Women & $\%$ \\
\hline \multicolumn{9}{|l|}{ IPSA } \\
\hline 2 & 0 & 0 & 9 & 0 & 0 & 3 & 0 & 0 \\
\hline \multicolumn{9}{|l|}{ APSA } \\
\hline 14 & 4 & 28.6 & 65 & 12 & 18.5 & 21 & I & 4.8 \\
\hline \multicolumn{9}{|c|}{$\begin{array}{l}\text { Judicial Studies } \\
\text { Committee 9; Section } 17 \text { and } \\
\text { on Law, Courts, and Judicial Process) }\end{array}$} \\
\hline \multicolumn{3}{|c|}{ Chairpersons } & \multicolumn{3}{|c|}{ Paper Givers } & \multicolumn{3}{|c|}{ Discussants } \\
\hline Total & Women & $\%$ & Total & Women & $\%$ & Total & Women & $\%$ \\
\hline \multicolumn{9}{|l|}{ IPSA } \\
\hline 2 & 0 & 0 & 8 & 1 & 12.5 & 4 & 1 & 25.0 \\
\hline \multicolumn{9}{|l|}{ APSA } \\
\hline 20 & 3 & 15.0 & 77 & 14 & 18.2 & 26 & 6 & 23.1 \\
\hline
\end{tabular}


Science and Politics

(Research Committee 1: Organized Section on Science and Technology Studies)

\begin{tabular}{|c|c|c|c|c|c|c|c|c|}
\hline \multicolumn{3}{|c|}{ Chairpersons } & \multicolumn{3}{|c|}{ Paper Givers } & \multicolumn{3}{|c|}{ Discussants } \\
\hline Total & Women & $\%$ & Total & Women & $\%$ & Total & Women & $\%$ \\
\hline IPSA & & & & & & & & \\
\hline 2 & 0 & 0 & 10 & 0 & 0 & 6 & 0 & 0 \\
\hline APSA & & & & & & & & \\
\hline 4 & 0 & 0 & 16 & 3 & 18.1 & 7 & I & 14.3 \\
\hline
\end{tabular}

(Research Committee 12; Organized Section on Politics and the Life Sciences)

\begin{tabular}{|c|c|c|c|c|c|c|c|c|}
\hline \multicolumn{3}{|c|}{ Chairpersons } & \multicolumn{3}{|c|}{ Paper Givers } & \multicolumn{3}{|c|}{ Discussants } \\
\hline Total & Women & $\%$ & Total & Women & $\%$ & Total & Women & $\%$ \\
\hline \multicolumn{9}{|l|}{ IPSA } \\
\hline 3 & 0 & 0 & 11 & 3 & 27.3 & 9 & 4 & 44.4 \\
\hline \multicolumn{9}{|l|}{ APSA } \\
\hline 5 & 0 & 0 & 16 & 1 & 6.3 & 12 & 2 & 16.7 \\
\hline \multicolumn{9}{|c|}{$\begin{array}{c}\text { Executives } \\
\text { (Study Group 28; Section } 14 \text { and Organized Section on Presidency Research) }\end{array}$} \\
\hline \multicolumn{3}{|c|}{ Chairpersons } & \multicolumn{3}{|c|}{ Paper Givers } & \multicolumn{3}{|c|}{ Discussants } \\
\hline Total & Women & $\%$ & Total & Women & $\%$ & Total & Women & $\%$ \\
\hline \multicolumn{9}{|l|}{ IPSA } \\
\hline 3 & 0 & 0 & 11 & 3 & 27.3 & 3 & 1 & 33.3 \\
\hline \multicolumn{9}{|l|}{ APSA } \\
\hline 9 & 1 & 11.1 & 36 & 5 & 13.9 & 13 & 1 & 17.7 \\
\hline \multicolumn{9}{|c|}{$\begin{array}{l}\text { International Economy } \\
\text { (Research Committee 17; Section 26) }\end{array}$} \\
\hline \multicolumn{3}{|c|}{ Chairpersons } & \multicolumn{3}{|c|}{ Paper Givers } & \multicolumn{3}{|c|}{ Discussants } \\
\hline Total & Women & $\%$ & Total & Women & $\%$ & Total & Women & $\%$ \\
\hline \multicolumn{9}{|l|}{ IPSA } \\
\hline 2 & 0 & 0 & 9 & 0 & 0 & 1 & 0 & 0 \\
\hline \multicolumn{9}{|l|}{ APSA } \\
\hline 8 & 4. & 50.0 & 31 & 6 & 19.4 & 7 & 0 & 0 \\
\hline
\end{tabular}

\section{Federalism}

(Research Committee 28; Section 15 and Organized Section on Federalism)

\begin{tabular}{|c|c|c|c|c|c|c|c|c|}
\hline \multicolumn{3}{|c|}{ Chairpersons } & \multicolumn{3}{|c|}{ Paper Givers } & \multicolumn{3}{|c|}{ Discussants } \\
\hline Total & Women & $\%$ & Total & Women & $\%$ & Total & Women & $\%$ \\
\hline \multicolumn{9}{|l|}{ IPSA } \\
\hline 3 & 0 & 0 & 10 & 1 & 5.6 & 3 & 0 & 0 \\
\hline \multicolumn{9}{|l|}{ APSA } \\
\hline 7 & 0 & 0 & 5 & 6 & 11.3 & 11 & 3 & 27.3 \\
\hline
\end{tabular}


Religion and Politics

(Study Group 25; Organized Section on Religion and Politics)

\begin{tabular}{|c|c|c|c|c|c|c|c|c|}
\hline \multicolumn{3}{|c|}{ Chairpersons } & \multicolumn{3}{|c|}{ Paper Givers } & \multicolumn{3}{|c|}{ Discussants } \\
\hline Total & Women & $\%$ & Total & Women & $\%$ & Total & Women & $\%$ \\
\hline IPSA & & & & & & & & \\
\hline 3 & 0 & 0 & 14 & 2 & 14.3 & - & - & - \\
\hline APSA & & & & & & & & \\
\hline 12 & 0 & 0 & 41 & 4 & 9.8 & 16 & 1 & 6.2 \\
\hline
\end{tabular}

IPSA has a Research Committee on Sex Roles and Politics, and a Study Group on Women, Politics, and Development to bring together female and male scholars interested in advancing investigation of these subjects. What is lacking is a women's caucus to provide a network to give aid and comfort to women political scientists whether or not they have a research interest in the status of women and to lobby IPSA towards greater parity. 